\title{
Cirugía de colon abierta con "Fast Track" o recuperación acelerada*
}

\author{
Dr. LEONARDO ESPÍNDOLA S. ${ }^{1}$
}

1 Servicio de Cirugía. Hospital Militar, Santiago, Chile.

\section{TRABAJO DE INGRESO}

\section{Abstract}

\section{"Fast Track" large bowel surgery}

Introduction: Large Bowel resective surgery requires 6 to 10 days in-hospital stay. Recently the multimodality rehabilitation concept or "fast track" surgery, a combination of different techniques in order to reduce surgical stress, increase recuperation and decrease in-hospital stay, has become an option. Objective: Evaluate postoperative evolution of patients submitted for elective large bowel and rectum resection using "Fast Track" protocol. Material and Method: Prospective Fast track protocol includes all patients submitted for elective large bowel and rectum resection in Military Hospital of Santiago during August 2004 to November 2005. Outcomes concerning postoperative ileus, in-hospital stay, complications and patient satisfaction were recorded. Results: 40 patients, 19 male (48\%) and 21 female (52\%) consecutive patients were included. Average age 66 years (39-87), 24 patients (60\%) were classified ASA II and 38\% ASA I. During the first 48 hrs $97.5 \%$ had anal flatulence. First defecation occurred before 48 hrs in $47.5 \%$ and in $37.5 \%$ at $72 \mathrm{hrs}$. Hospital discharge at $4^{\text {th }}$ day was possible in $78 \%$. Pain visual analogical scale at $24 \mathrm{hrs}$ was less than 2 in $95 \%$. Satisfaction rate was very good in $70 \%$. Morbidity was $18 \%$ without mortality. Anastomotic dehiscence was $2.5 \%$. Conclusions: Fast Track protocol reduces in- hospital stay, pain and postoperative ileus.

Key words: Large bowel surgery, "Fast Track" surgery, rehabilitation, perioperative care.

\section{Resumen}

Introducción: La Cirugía resectiva de colon se asocia a una hospitalización de 6 a 10 días. En el último tiempo se ha desarrollado el concepto de rehabilitación multimodal o "Fast track" que involucra una combinación de técnicas que llevan a reducir la respuesta al stress quirúrgico, optimizar la recuperación y reducir los días de hospitalización. Objetivo: Evaluar el postoperatorio de pacientes sometidos a cirugía electiva de colon y recto superior utilizando un protocolo de "Fast track". Material y Método: Ingresaron a un protocolo prospectivo de recuperación acelerada, todos los enfermos sometidos a cirugía electiva de colon y recto superior en el Hospital Militar de Santiago en el período comprendido entre Agosto 2004 a Noviembre 2005. Se evalúa íleo postoperatorio, días de hospitalización, complicaciones postoperatorias y satisfacción de pacientes. Resultados: Ingresaron 40 pacientes consecutivos, 19 hombres (48\%) y 21

\footnotetext{
*Recibido el 30 de Mayo de 2007 y aceptado para publicación el 6 de Octubre de 2008.
}

Correspondencia: Dr. Leonardo Espíndola S.

Fernandez Mira 796, Santiago, Chile.

E-mail: Leo2183@vtr.net 
mujeres (52\%). La edad promedio fue 66 años (39-87), 24 pacientes (60\%) eran ASA II y 38\% ASA I. El 97,5\% expulsó gases en las primeras $48 \mathrm{~h}$. La primera defecación ocurrió dentro de las primeras $48 \mathrm{~h}$ en el $47,5 \%$ de los pacientes y en el 37,5\% a las $72 \mathrm{~h}$. El $78 \%$ de los paciente fueron dados de alta al $4^{\circ}$ día. La escala visual análoga (EVA) para dolor a las 24 h era $<2$ en el 95\%. Grado de satisfacción muy bueno en el $70 \%$. Morbilidad de $18 \%$ y sin mortalidad. Dehiscencia de anastomosis fue $2,5 \%$. Conclusiones: El protocolo de recuperación acelerada reduce la estadía hospitalaria, disminuye el dolor e íleo post-operatorio.

Palabras clave: Cirugía de colon, cirugía con "fast track", rehabilitación, manejo perioperatorio.

\section{Introducción}

La Cirugía de colon usualmente se asocia a una tasa de complicaciones de $15-20 \%$ y una hospitalización post-operatoria de 6 a 10 días ${ }^{1-5}$. Factores limitantes para un alta precoz son dolor, vómitos, náuseas, íleo postoperatorio, disfunción de órganos por stress, fatiga y factores tradicionales tales como uso de sonda nasogástrica, drenajes, etc, los cuales retardan la recuperación y contribuyen a la morbilidad. En años recientes numerosos avances en técnica quirúrgica y manejo postoperatorio ha resultado en estadías de hospitalización más breves y mejoramiento en la función fisiológica de los pacientes sometidos a cirugía de colon.

En el último tiempo se ha publicado una serie de trabajos en los cuales se ha desarrollado el concepto de rehabilitación multimodal postoperatoria o "Fast track", liderado principalmente por el grupo del Dr. Kehlet en Dinamarca ${ }^{6,7}$. Este concepto involucra una combinación de técnicas con el objetivo de reducir la respuesta al stress quirúrgico, optimizar la recuperación y reducir los días de hospitalización. Los métodos usados incluyen anestesia peridural torácica, agresiva movilización postoperatoria y realimentación precoz. Este manejo de los pacientes sometidos a cirugía abierta de colon está asociado a una recuperación precoz de la función intestinal, disminución de la respuesta al stress, mejoramiento en la función física y reducción de la estadía hospitalaria.

El objetivo de este trabajo es evaluar el postoperatorio de pacientes sometidos a cirugía electiva abierta de colon y recto superior utilizando un protocolo de "Fast Track" desde el punto de vista del dolor postquirúrgico, íleo, complicaciones postoperatorias, estadía hospitalaria y satisfacción del paciente.

\section{Material y Método}

Ingresaron a este protocolo prospectivo de manejo, todos los enfermos sometidos a cirugía electiva abierta de colon y recto superior que se operaron en el equipo de coloproctología del Hospital Militar de Santiago durante el período de tiempo comprendido entre agosto 2004 a Noviembre 2005 y con su consentimiento informado. Se excluyeron de este protocolo los pacientes con cirugía de recto medio e inferior, los pacientes que quedarían con ostomías y las colectomías totales.

Todos los pacientes se clasificaron según sus comorbilidades de acuerdo a los criterios de la Sociedad Americana de Anestesiólogos (ASA I, II o III).

\section{Manejo pre-operatorio}

- Hospitalización 24 h previo a la cirugía.

- Preparación mecánica sólo para las cirugías del colon izquierdo y recto.

- Antibióticos profilácticos 1 hora antes de la cirugía:

Ciprofloxacino 200 mgr IV

Metronidazol 500 mgr IV

- Sin premedicación anestésica.

\section{Analgesia y anestesia}

- A todos los pacientes se les coloca catéter peridural, insertado a nivel T6-T7 para hemicolectomía derecha y a nivel de T8-T10 para hemicolectomía izquierda, sigmoidectomía y resección de recto.

- Se utiliza dosis de prueba con lidocaína 1\% 20 $\mathrm{mg}$ y durante el intraoperatorio se aplican bolos de bupivacaína $0,25 \% 10$ a $15 \mathrm{mg}$.

- Todos fueron sometidos a anestesia general con intubación endotraqueal

- Se mantuvo la normotermia durante la anestesia.

- Infiltración de la aponeurosis con bupivacaína al $0,25 \%$ al finalizar la cirugía.

\section{Intraoperatorio}

- Incisión transversa $2 \mathrm{~cm}$ sobre ombligo para hemicolectomía derecha. Cirugía del lado izquierdo con laparotomía media.

- Anastomosis manual o con stapler en hemicolectomías derechas y con sutura mecánica en las del lado izquierdo.

\section{Manejo postoperatorio:}

- El postoperatorio se realiza las primeras dos horas en recuperación, posteriormente en el intermedio quirúrgico por $24 \mathrm{~h}$. 
- No está indicado el uso sonda nasogástrica.

- Para la analgesia, que se realiza a través del catéter peridural, infusión de mezcla de bupivacaína $0,1 \%$ y Fentanyl $2 \mathrm{Gama} / \mathrm{ml}$ a $5 \mathrm{ml}$ hora, en caso de necesidad de refuerzo analgésico aumentar infusión a $7 \mathrm{ml} / \mathrm{h}$.

- Además se indica ketoprofeno 100 mg cada 8 horas EV

- No usamos opiáceos o derivado alguno de la morfina EV.

- Idon $^{\mathrm{R}} 1$ ampolla c/8 IV hasta el día del alta. Al tercer día por vía oral.

- Omeprazol en dosis de $40 \mathrm{mg}$ al día durante todo el postoperatorio.

- Heparina de bajo peso molecular.

- Catéter peridural removido a las $48 \mathrm{~h}$ del postoperatorio por anestesista.

- Se retira sonda Foley a las 48 h.

- Se moviliza y levanta al paciente durante 2 horas, a las 6 horas del post-operatorio.

- Régimen líquido fraccionado, Volumen total 1.000 $\mathrm{ml}$, a las 10 horas del postoperatorio.

- Movilizar más de 8 horas en el segundo día postoperatorio.

- Régimen líquido volumen total 1.500.

- Régimen líquido amplio volumen libre al $2^{\circ}$ día postoperatorio.

- Régimen liviano al $3^{\text {er }}$ día postoperatorio.

- Paracetamol 500 mg c/6 h vía oral desde primer día postoperatorio y por 6 días.

- Sulfato de mg $10 \mathrm{gr}$ al $4^{\text {to }}$ día postoperatorio si no hay defecación.

\section{Objetivos a evaluar}

- Presencia de íleo postoperatorio (primera defecación).

- Número de días de estadía.

- Complicaciones postoperatorias.

- Satisfacción de pacientes.

- Dolor post-quirúrgico según escala visual análoga (EVA) a las 2, 4, 6, 12, 48, 72 horas.

\section{Resultados}

Se estudió con este protocolo prospectivo 40 pacientes consecutivos, entre Agosto 2004 y Noviembre 2005, los que fueron operados en forma electiva, por cirugía abierta de colon y recto superior. Todos los enfermos cumplieron con los criterios de inclusión para el protocolo de "Fast track". Del total de pacientes ingresados al protocolo 19 fueron hombres (48\%) y 21 fueron mujeres (52\%). La edad promedio del grupo fue 66 años con un rango entre 39 a 87 años. El score preoperatorio de la sociedad americana de anestesiología (ASA), para evaluar riesgo quirúrgico mostró que 24 pacientes (60\%) eran ASA II, 15 enfermos (38\%) correspondían a ASA I y $2 \%$ ASA III.

Las cirugías realizadas pueden verse en la Figura 1 , siendo las más frecuentes las hemicolectomías derecha e izquierdas y sigmoidectomías. En la Figura 2 se pueden observar las patologías médicas asociadas más frecuentes, y los antecedentes quirúrgicos en la Tabla 1.

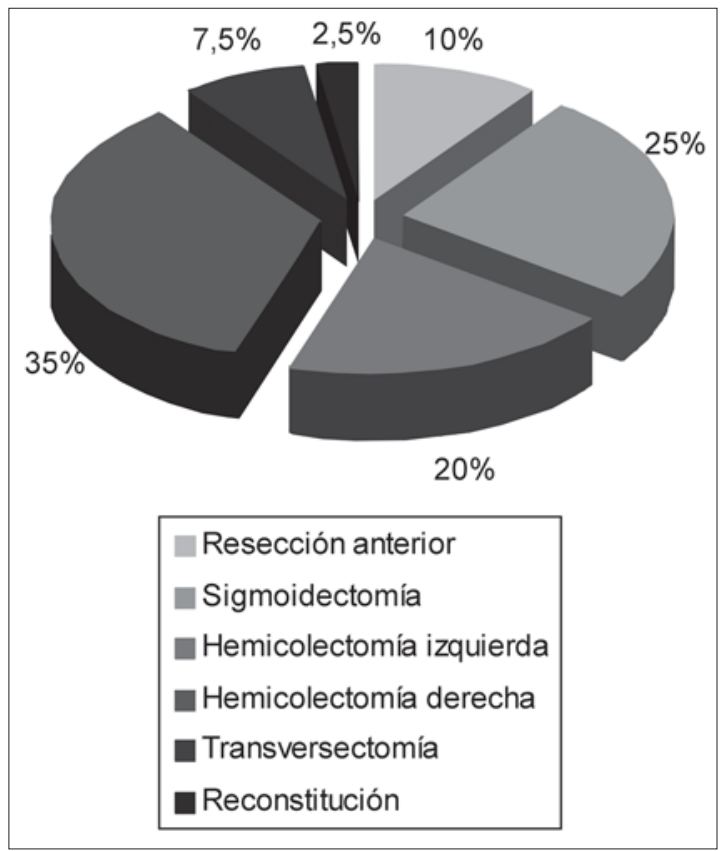

Figura 1. Tipo de cirugía.

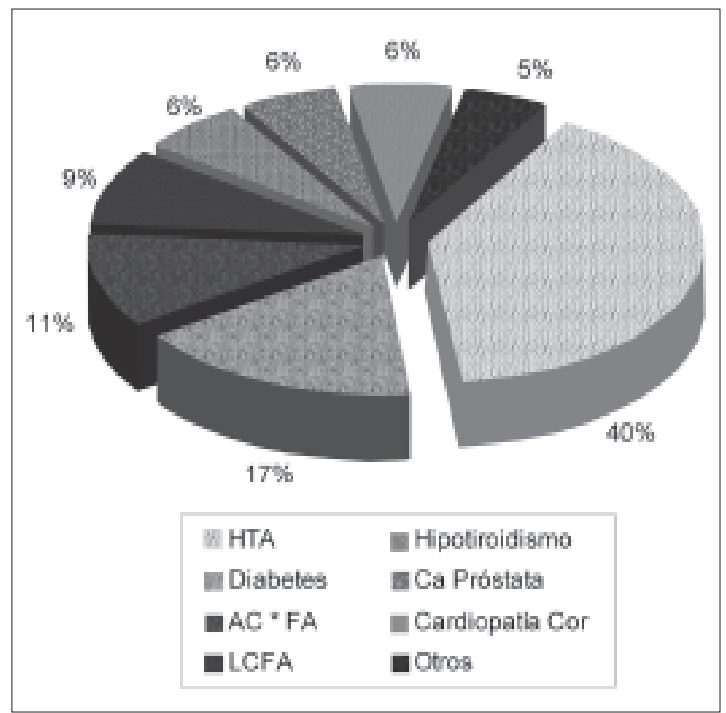

Figura 2. Comorbilidades. 
Con respecto al transito intestinal relacionado a la expulsión de gases, el 47,5\% (19 enfermos) lo tuvo dentro de las primeras $24 \mathrm{~h}, 20$ pacientes $(50 \%)$ a las $48 \mathrm{~h}$ y sólo un enfermo a las $72 \mathrm{~h}$. La primera defecación en nuestro estudio mostró que el $5 \%$ de los enfermos fue en las primeras $24 \mathrm{~h}$, el $42,5 \%$ a las $48 \mathrm{~h}, 37,5 \%$ de los pacientes a las $72 \mathrm{~h}$ y un $5 \%$ del grupo estudiado al $4^{\circ}$ día.

La estadía hospitalaria de los pacientes, que se presenta en la Figura 3, muestra que el 93\% de los enfermos fueron dados de alta en forma precoz: al $4^{\circ}$ día 31 pacientes (78\%) y al quinto día el $15 \%$.

Complicaciones menores, las cuales fueron transitorias y no alteraron el resultado final, se presentan en 24 pacientes; corresponden a hipotensión en 10 enfermos (25\%) lo cual es atribuible a la peridural torácica, náuseas en 7 pacientes, vómitos en 3 pacientes, sangrado y fiebre en 2 pacientes respectivamente. Las complicaciones mayores pueden verse en la Tabla 2.

Tabla 1. Antecedentes quirúrgicos

\begin{tabular}{llr}
\hline Colecistectomía & 6 pacientes & $(15 \%)$ \\
Apendicectomía & 3 pacientes & $(8 \%)$ \\
Aneurisma aorto-ilíaco & 1 paciente & \\
Gastrectomía & 1 paciente \\
Pancreatoduodenectomía & 1 paciente \\
\hline
\end{tabular}

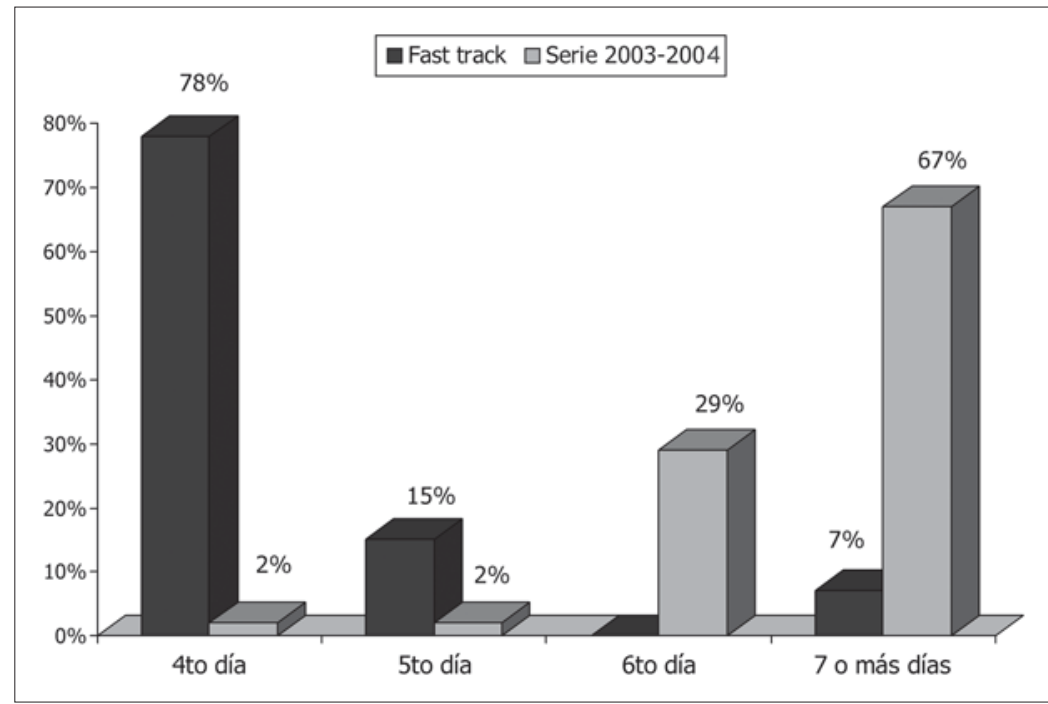

Figura 3. Estadía hospitalaria.
La medición del dolor basado en la escala visual análoga (EVA) reveló que la mayoría de los pacientes casi no tienen dolor o es muy leve $(\mathrm{EVA}<2)$ durante la hospitalización. Durante las primeras 12 $\mathrm{h}$ el $95 \%$, un total de 38 enfermos, presenta un EVA menor a 1. La misma situación se observa a las 24 horas post-cirugía en la cual el $95 \%$ de los pacientes también presenta EVA menor 1 (Figura 4). A las 48 y $72 \mathrm{~h}$ postoperatorias el número de pacientes con EVA $<1$ es un $65 \%$ y $30 \%$ de los enfermos presentan EVA $<3$.

Otro de los parámetros evaluados fue el grado de satisfacción de los pacientes, a los cuales se les preguntaba en el primer control ambulatorio. Los resultados muestran que el $70 \%$ de los pacientes tenían un grado de satisfacción muy bueno, $23 \%$ bueno y el $7 \%$ regular. Ningún paciente tuvo un grado de satisfacción malo. Durante el período de tiempo de este protocolo hubo 2 reingresos $(5 \%)$, ninguno de los cuales necesito de cirugía. El primer

\section{Tabla 2. Complicaciones postoperatorias}

\begin{tabular}{lcc}
\hline & $\mathbf{n}$ & $\mathbf{\%}$ \\
\hline Mortalidad & 0 & 0 \\
Morbilidad & 18 & \\
Dehiscencia anastomosis & 2,5 & 1 \\
Necrosis Intestinal & 2,5 & 1 \\
Hemoperitoneo & 5 & 2 \\
Infección herida operatoria & 5 & 2 \\
Seroma herida & 2,5 & 1 \\
\hline
\end{tabular}




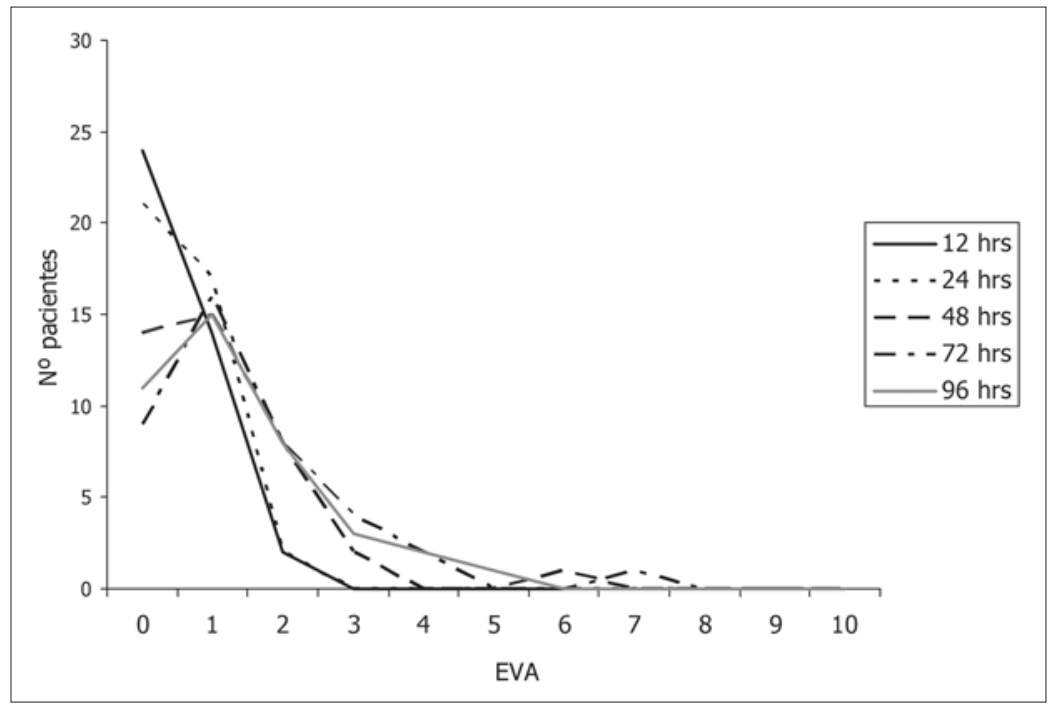

Figura 4. Dolor postoperatorio.

paciente se rehospitalizó por vómitos debido a cuadro de gastritis diagnosticada por endoscopia y se manejo sólo con terapia médica. El segundo paciente presentó cuadro febril a los doce días postoperatorios y se manejo con antibioterapia. No se supo la causa del cuadro febril.

\section{Discusión}

La estadía hospitalaria post-cirugía abierta de colon y recto electiva ha sido entre 5-7 días con una mediana de 7 días $^{8-10}$. Algunos estudios, recientes, muestran una estadía más prolongada con un promedio de 11,3 días para la cirugía colorrectal con complicaciones y de 6,5 días para aquella sin complicaciones ${ }^{11}$.

Durante los últimos años se ha cuestionado el manejo postoperatorio tradicional de los pacientes sometidos a este tipo de cirugía debido al mayor conocimiento de la fisiología postoperatoria y al avance en la terapia intensiva. El factor clave patogénico en la morbilidad postoperatoria, excluyendo complicaciones de la técnica quirúrgica o anestésica, es la respuesta al stress quirúrgico que induce un aumento en la demanda de los órganos mediante cambios bioquímicos y endocrinos ${ }^{12-18}$. Varias técnicas se han desarrollado en el último tiempo con el fin de atenuar esta disfunción de órganos postoperatorios y así disminuir la morbilidad asociada. Entre las técnicas desarrolladas se destacan anestesia peridural torácica, movilización postoperatoria agresiva y realimentación precoz. Hay que consignar que un factor limitante para este manejo postoperatorio de recuperación acelerada o "Fast track", son algunas tradiciones quirúrgicas heredadas, algunas sin una sólida base científica y entre las cuales destacan el uso de drenajes, sondas nasogástricas, restricciones para la movilización, limitaciones en la ingesta oral, etc.

Numerosa evidencia científica actual, demuestra que el uso de sonda nasogástrica no es necesaria, la sonda Foley puede usarse por períodos cortos de tiempo (1-2 días), y la alimentación precoz puede ser de rutina sin riesgos ${ }^{16,17}$. Esto ha llevado a que un mayor número de cirujanos aceptan actualmente que la estadía postquirúrgica puede ser reducida en forma segura ${ }^{12,13}$.

También hay que mencionar el impacto que ha significado la cirugía laparoscópica colorrectal debido a que ha sido capaz de disminuir el íleo postoperatorio, con alimentación precoz y reducción en la estadía intrahospitalaria en pacientes con anastomosis intestinal $1^{14,15}$.

Numerosos trabajos publicados en la literatura en años recientes, que han utilizado protocolos de "Fast track", confirman que la hospitalización puede ser reducida en cirugía colorrectal abierta sin aumentar la morbilidad y las complicaciones ${ }^{18-20}$. Uno de los factores limitantes en la cirugía abierta colorectal es el íleo intestinal postoperatorio que puede reducirse en forma significativa con el uso de anestesia peridural con anestésicos locales, como ha sido señalado por numerosos trabajos en los últimos años. La anestesia peridural a nivel torácico, dependiendo del lugar de la incisión (T7-T8, T8-T9, T9-T10) produce una disminución de la respuesta del organismo al stress por inhibición del simpáti- 
co, reduciendo las complicaciones cardíacas, pulmonares e íleo postoperatorio; la movilización y la alimentación precoz también tendrían un rol importante en la recuperación rápida del tránsito intestinal $^{21-26}$

El presente estudio prospectivo utilizando un protocolo de "Fast track" o recuperación acelerada en pacientes sometidos a cirugía electiva de colon y recto muestra que una recuperación precoz de la función de los órganos puede ser obtenida. En nuestra serie el $97 \%$ de los enfermos presentó transito intestinal para gases en las primeras $48 \mathrm{~h}$ y el $87 \%$ de los pacientes tuvo la primera defecación entre el $2^{\circ}$ y $3^{\text {er }}$ día. Estos resultados concuerdan con los reportes en la literatura en el sentido que una anestesia peridural combinada con movilización precoz y alimentación en el postoperatorio inmediato reducen el íleo postcirugía abierta de colon, siendo un hecho relevante. El rol de los prokinéticos intestinales en evitar el íleo no ha sido demostrado en trabajos prospectivos pero jugarían un papel importante debido a la etiología multifactorial de la parálisis intestinal ${ }^{21}$.

$\mathrm{Al}$ analizar nuestra serie de pacientes operados de cirugía de colon y recto, no publicada, utilizando los mismos criterios de inclusión de este protocolo (Tabla 3) pero sin un protocolo de "Fast track", el $73 \%$ de los enfermos presentaba la primera defecación entre el $6^{\circ}$ y $7^{\circ}$ día. En la literatura nacional el Dr. López y su grupo en un estudio comparativo entre cirugía laparoscópica y convencional para enfermedad diverticular refiere, para esta última, un retorno de la actividad intestinal al $4^{\circ}$ día para gases y al $7^{\circ}$ día para deposiciones datos que son claramente mayores que nuestra serie, incluso los resultados obtenidos para su protocolo de abordaje laparoscópico son similares a nuestro estudio ${ }^{27}$.

En relación a la estadía intrahospitalaria, en nuestra serie el $78 \%$ de los enfermos fue dado de alta al $4^{\circ}$ día y un $15 \%$ al $5^{\circ}$, es decir que menos del $10 \%$ de los enfermos estuvieron 7 ó más días. Al analizar la serie de enfermos operados previos a la realización del protocolo el $29 \%$ fue dado de alta al $6^{\circ}$

Tabla 3. Características años 2003-2004 (Sin protocolo de "Fast Track")

\begin{tabular}{lll}
\hline 42 pacientes: & Edad promedio & 67 años (19-86) \\
& Hombres 45 \% & Mujeres 55 \% \\
Tipo de cirugía: & Hemicolectomía izquierda & $19 \%$ \\
& Sigmoidectomia & $28 \%$ \\
& Resección Anterior & $28 \%$ \\
& Hemicolectomía derecha & $29 \%$ \\
Mortalidad & $0 \%$ \\
Morbilidad & $17 \%$ \\
\hline
\end{tabular}

día, el $67 \%$ estuvo hospitalizado 7 ó más días, y sólo el 4\% (Figura 4) permaneció en el hospital entre 4 a 5 días. El Dr. Manterola en una revisión del 2005 refiere una hospitalización de 10 días para cirugía abierta y de 6 días para la laparoscópica ${ }^{28}$. Trabajos publicados en Chile por el grupo del Dr. Bannura muestran una estadía intrahospitalaria de 9,8 días para pacientes sometidos a cirugía de colon abierto en forma electiva por enfermedad diverticular $^{29}$. Boza, en su publicación en relación al tratamiento quirúrgico electivo del cáncer de colon, refiere un promedio de hospitalización de 12 días $^{30}$. Bannura refiere hospitalizaciones de 11 días para la cirugía de colon por cáncer ${ }^{31}$; el reporte del Dr. López es de 10 días para la cirugía convencional. Debo señalar que la estadía hospitalaria para cirugía laparoscópica publicada por este último grupo es de 4 días siendo similar a esta serie presenta$\mathrm{da}^{27}$. Los reportes en la literatura utilizando protocolos de " Fast Track" similares a este protocolo incluso presentan estadías más abreviadas, dos días, como en la mayoría de los trabajos presentados por Kehlet y su grupo ${ }^{6,8}$.

Un dato relevante en nuestro protocolo fue el dolor postoperatorio que en la mayoría de los pacientes fue EVA entre 0 y 3 , considerado leve, gracias a la peridural torácica. Esto permitió la deambulación precoz de los enfermos, un menor stress asociado y alimentación precoz.

Un hecho que se observó en los pacientes mayores a 75 años que se sometieron a cirugía de colon y recto a este protocolo de recuperación acelerada, es que no presentaron episodios de agitación psicomotora y desorientación, variable no evaluada por este por este estudio y que necesita de nuevos trabajos prospectivos y randomizados. Algo similar se observa con la tasa de complicaciones cardiopulmonares las cuales no se presentaron en esta serie, hecho también consignado en los trabajos de Kehlet et al. Esto se explica por una menor respuesta al stress quirúrgico, debido a la anestesia peridural y menor administración de fluidos endovenosos ${ }^{6,8}$. Al evaluar los resultados desde el punto de vista de morbilidad, mortalidad y dehiscencia de anastomosis no presentan diferencias con lo reportado clásicamente en la literatura. En nuestra serie no hubo mortalidad durante el período estudiado y el porcentaje de filtración de la anastomosis fue de un $2,5 \%$, valor dentro de lo esperado en centros dedicados a la cirugía colo-rectal ${ }^{27-30}$.

Hay que recalcar que los criterios utilizados en este protocolo de estudio, control adecuado del dolor, tránsito intestinal y tolerancia a la ingesta de sólidos para dar de alta a los pacientes, son los mismos que se utilizan tradicionalmente pero logrados en un período de tiempo más breve. 
Los resultados de este estudio prospectivo, junto a la gran cantidad de trabajos publicados en la literatura de protocolos de recuperación acelerada, dentro del contexto de discusión entre cirugía abierta o laparoscópica en colon, muestran que la recuperación más breve atribuida a esta última técnica quirúrgica (menos dolor postoperatorio, alta precoz y menos incidencia de íleo) no es diferente sino más bien $\operatorname{similar}^{23}$. Si se observan los resultados presentados por el grupo de la U. Católica en relación al abordaje laparoscópico electivo en la enfermedad diverticular, vemos que no hay diferencias a lo presentado por este protocolo de "Fast Track"27. Este mismo grupo en una publicación más reciente, donde muestran su experiencia en cirugía colorrectal por vía laparoscópica, refieren una mediana de hospitalización de 5 días y tránsito intestinal para gases de dos días, resultados similares a lo mostrado en este estudio ${ }^{32}$. Esto sugeriría que la recuperación postoperatoria depende de factores tales como alivio del dolor en forma óptima, alimentación y movilización precoz y al no uso de drenajes, sondas nasogástricas, catéter urinario prolongado, más que a la elección de la técnica quirúrgica en si misma (laparoscópica o abierta) ${ }^{24}$. Estudios más recientes, que comparan estas dos técnicas quirúrgicas, en trabajos prospectivos, doble ciego y randomizados, revelan que no existirían diferencias en la estadía hospitalaria y tiempo de recuperación en relación a la cirugía de colon ${ }^{25,26}$. Sin embargo, se plantea la necesidad de un estudio prospectivo en nuestra unidad para confirmar esta apreciación.

\section{Conclusiones}

- El protocolo de recuperación acelerada o "Fast track":

- Reduce la estadía hospitalaria.

- Disminuye el dolor post-operatorio.

- Disminuye el Íleo post-quirúrgico.

- No existen diferencias en porcentaje de dehiscencia de anastomosis y mortalidad operatoria con la cirugía tradicional.

- Los resultados obtenidos por este protocolo recomiendan un protocolo de "Fast track" o recuperación acelerada a todos los pacientes sometidos a cirugía abierta de colon y recto operados en forma electiva.

\section{Referencias}

1. Bokey EL, Chapuis PH, Fung C. Postoperative morbidity and mortality following resection of the colon and Rectum for cancer. Dis Colon Rectum 1995; 38: 480-487.

2. Retchin SM, Penberty L, Brown R Clement D. Perioperative management of colon cancer under Medicare risk programs. Arch Intern Med 1997; 157: 1878-1884.

3. Colorectal cancer Collaborative Group: Surgery for colorectal cancer in Elderly patient: a systematic review. Lancet 2000; 356: 968-974.

4. Seanogore AJ, Delaney CP, Brady KM, Fazio VW. Results of a standardized technique and postoperative care plan for laparoscopic sigmoid colectomy: a 30month experience. Dis Colon Rectum 2003; 46: 503509.

5. Senagore AJ, Madbouly KM, Fazio VW, Delaney CP. Advantages of laparoscopic colectomy in older patient. Arch Surg 2003; 138: 252-256.

6. Kehlet H, Wilmore DW, Multimodal strategies to improve surgical outcome. Am J Surg 2002; 183: 630641.

7. Wilmore DW, Kehlet H. Management of patients in fast track surgery. BMJ 2001; 322: 473-476.

8. Kehlet H. Multimodal approach to control postoperative pathophysiology and rehabilitation. $\mathrm{Br} \mathrm{J}$ Anaesth 1997; 78: 606-617.

9. Schoetz DJJ, Bockler M, Rosenblatt MSl. 'Ideal' length of stay after colectomy: whose ideal? Dis Colon Rectum 1997; 40: 806-810.

10. Bokey EL, Chapuis PH, Fung C. Postoperative morbidity and mortality following resection of the colon and rectum for cancer. Dis Colon Rectum 1995; 38: 480-487.

11. Retchin SM, Pinberthy L, Desch C. Perioperative management of colonic cancer under medicare risk programs. Arch Int Med 1997; 157: 1878-1884.

12. Pearson SD, Goulart-Fisher D, Lee TH. Critical pathways as a strategy for improving patient care. Ann Intern Med 1995; 123: 941-948.

13. Archer SB, Burnett RJ, Flesch LV. Implementation of a clinical pathway decreases length of stay and hospital charges for patients undergoing total colectomy and ileal pouch/anal anastomosis. Surgery 1997; 122: 699-705.

14. Bardram L, Funch-Jensen P, Jensen P. Recovery after laparoscopic colonic surgery with epidural analgesia and early oral nutrition and mobilization. Lancet 1995; 345: 763-764.

15. Senagore AJ, Luchtefeld MA, MacKeigan J. What is the learning curve for laparoscopic colectomy? Am Surg 1995; 61: 681-685.

16. Kehlet H. Multimodal approach to control postoperative pathophysiology and rehabilitation. $\mathrm{Br} \mathrm{J}$ Anaesth 1997; 78: 506-517.

17. Kehlet H, Wilmore DW. Multimodal strategies to improve surgical outcome. Am J Surg 2002; 183: 63041. 
18. Bradshaw B, Spencer S, Thirlby R. Standard perioperative care protocols and reduced length of stay after colon surgery. J Am Coll Surg 1998; 186: 501-506.

19. Di Fronzo L, Cymerman J. O'Connell T. Factors affecting early postoperative feeding following elective open colon resection. Arch Surg 1999; 134: 941-945.

20. Senagore AJ, Duepree HJ, Delaney CP, Dissanaike S, Brady KM, Fazio VW. Cost structure of laparoscopic and open sigmoid colectomy for diverticular disease: similarities and differences. Dis Colon Rectum 2002; 45: 485

21. Holte K, Kehlet H. Postoperative ileus: a preventable event. Br J Surg 2000; 87: 1480-1493.

22. Carli F, Trudel JL, Belliveau P. The effect of intraoperative thoracic epidural anesthesia and postoperative analgesia on bowel function after colorectal surgery: A prospective randomized trial. Dis Colon Rectum 2001; 44: 1083-1089.

23. Chapman AE, Levitt MD, Hewett P.Laparoscopic assisted resection of colorectal malignancies. A systematic review. Ann Surg 2001; 234: 590-606.

24. Basse L, Thorbøl J, Løssl K, Kehlet H. Colonic Surgery With Accelerated Rehabilitation or Conventional Care Ann Surg 2001; 232: 51-57.

25. Basse L, Jakobsen DH, Bardram L, Billesbølle P, Lund C, Mogensen T, et al. Functional Recovery after Open versus Laparoscopic Colonic Resection. A Randomized, Blinded Study. Ann Surg 2005; 241: 416-
423.

26. Basse L, Madsen JL, Billesbølle P. Gastrointestinal transit after laparoscopic vs open colonic resection. Surg Endosc. 2003; 17: 1919-1922.

27. López F, Soto G, Tapia G, Schnettler K, Zárate A, Avendaño R, y col. Cirugía laparoscópica electiva en enfermedad diverticular. Un estudio comparativo con la cirugía convencional. Rev Méd Chile 2003; 131: 719-726.

28. Manterola C, Pineda V, Vial M, Losada H, Muñoz S. Cáncer de colon. Elección de un tipo de terapia quirúrgica basado en la evidencia Revisión sistemática de la literatura. Rev Chil Cir 2005; 57: 220-228.

29. Bannura G, Contreras J, Melo C, Barrera A, Soto D, Mansilla J. Indicaciones resultados alejados del tratamiento quirúrgico electivo de la enfermedad diverticular del colon sigmoides Rev Méd Chile 2005; 133: 1037 1042.

30. Boza C, Zúñiga A, Rahmer A, López F. Cáncer de colon: Resultados del tratamiento quirúrgico electivo Rev Chil Cir 2000; 52: 629-634.

31. Bannura G, Cumsille M, Melo C, Barrera A, Contreras J. Tratamiento quirúrgico del cáncer de colon: Análisis de 120 pacientes consecutivos. Gastr Latinoam 2000; 11: 253-261.

32. López F. Cirugía laparoscópica colorrectal: análisis de 85 pacientes consecutivos. Rev Chil Cir 2004; 56: 255-262.

\section{INFORME TRABAJO DE INGRESO}

De las más de 80 millones de operaciones que cada año se realizan en los EE.UU., al menos un tercio son electivas y requieren tiempos de hospitalización que dependerán de los eventos postanestésicos y post-quirúrgicos que pueden ocurrir; y de los cuidados y manejos terapéuticos que obligarán a mantener al paciente hospitalizado. ¿Podemos hacer algo para modificar estos plazos logrando egresos hospitalarios seguros y más precoces? Sin duda que los avances en el manejo anestésico y perioperatorio de las dos últimas décadas han permitido disminuir estos requerimientos de hospitalización postoperatoria, pero para lograr los mejores resultados en este ámbito se hace necesario trabajar en la estandarización protocolizada para un manejo integral del paciente quirúrgico, con la aplicación de una variedad de técnicas que disminuirán las tasas de complicaciones, acortarán el período de convalecencia y disminuirá el uso de los recursos hospitalarios. Los tiempos han cambiado radicalmente desde la época de los años '70, cuando los pacientes que se someterían a una cirugía de colon ingresaban 5 días previos para limpiar el intestino, y los que no sufrían complicaciones mayores egresaban luego de 10 días de operados.

El trabajo de ingreso del Dr. Espíndola se refiere específicamente a pacientes sometidos a cirugía colorrectal electiva a los cuales se les aplica un protocolo de recuperación acelerada basado fundamentalmente en las experiencias comunicadas por el Dr. Henrik Kehlet en los últimos 9 años, y que tuvimos la oportunidad de conocer en nuestro reciente Congreso de Cirugía. El Dr. Kehlet apunta con énfasis a las áreas de intervención para lograr esta recuperación acelerada: la evaluación preoperatoria acuciosa para optimizar las disfunciones orgánicas cardíacas, pulmonares, metabólicas y otras; la correcta profilaxis antibiótica, la elección juiciosa de la técnica anestésica, la opción de procedimientos quirúrgicos mínimamente invasivos, la normotermia intraoperatoria, la evaluación de prácticas perioperatorias rutinarias y su correcta indicación; como el uso de sondas y drenajes, la realimentación y la deambulación precoces; completan estas medi- 
das la prevención de los tromboembolismos, de la náusea y el vómito y el control del dolor, que permitirán disminuir los tiempos de recuperación de las funciones básicas normales y que redundará en un egreso más precoz (que será una consecuencia, no un objetivo).

Como se podrá deducir, un pre-requisito básico para un programa de recuperación acelerada será la colaboración multidisciplinaria con una interacción activa del paciente, el cirujano, el anestesiólogo y los equipos de enfermería, kinesiología y nutrición.

El objetivo de este trabajo es la evaluación de sus resultados en cuanto a dolor, íleo, morbilidad, estadía hospitalaria y satisfacción del paciente.

En cuanto al manejo preoperatorio, el autor señala que se utilizó preparación mecánica sólo en las resecciones distales de colon y recto y no en las de colon derecho; al respecto, cada vez hay más evidencias que ponen en duda los beneficios de la preparación de rutina, y en base a ello nuestro grupo acaba de terminar un protocolo de uso versus no uso de preparación, con interesantes resultados que serán presentados en el curso de este año.

En relación a la anestesia y la analgesia, llama la atención que los catéteres peridurales hayan tenido sólo fines de analgesia postoperatoria, y que todos los pacientes hayan recibido anestesia general con intubación endotraqueal; no ha sido nuestra experiencia, ya que con frecuencia cada vez mayor hemos utilizado la anestesia peridural exclusiva en pacientes ancianos y/o con morbilidad asociada, sin lamentar por ello dificultades quirúrgicas.

No se utilizó sondaje intragástrico y el vesical fue retirado precozmente; estamos completamente de acuerdo con ello; algunas veces se requiere un sondaje gástrico intraoperatorio que es retirado al final de la cirugía. No se informa del uso de drenajes, por lo que suponemos que no tuvieron incidencia en los objetivos a alcanzar. En nuestra institución hemos optado por el uso de drenajes sólo en anastomosis pélvicas bajas, por lo que en las colectomías segmentarias no se requerirán salvo en situaciones de excepción.

El íleo postoperatorio es, junto con el dolor, el principal motivo de retraso en la recuperación de estos pacientes. Con el advenimiento de la cirugía laparoscópica se demostró una más rápida resolución del íleo en pacientes no laparotomizados; sin embargo, cabría preguntarse, como lo plantea el Dr. Espíndola, si es necesario esperar la reaparición de ruidos intestinales y/o la expulsión anal de gases para reiniciar la alimentación; o, expresado de otra forma, afectará la realimentación precoz la buena evolución de los pacientes sometidos a cirugía de colon por laparotomía? Durante décadas esta reingesta precoz fue considerada como un factor deletéreo y agravante del íleo postoperatorio, al crear un círculo vicioso de incremento de las secreciones endoluminales, distensión de asas y progresiva disfunción motora, elementos que en caso de resecciones con anastomosis podían predisponer a una falla de éstas por distensión e isquemia a nivel de la sutura. Varios autores en la década pasada, como Choi '96, Hawasi '96, Reissman '95 y Ortiz '96 desmitificaron esta idea al demostrar que la realimentación precoz no incrementa la frecuencia y duración del íleo, no aumenta las tasas de dehiscencia anastomótica y sí puede disminuir hasta en un $50 \%$ la estadía hospitalaria. Dentro de las intervenciones quirúrgicas que no parecen beneficiarse de una realimentación precoz están las colectomías totales y las proctocolectomías. En cuanto al uso de proquinéticos como Domperidona, Metoclopramida, Eritromicina, Cisaprida y otros, no hay evidencia que sustente su utilización rutinaria. Como bien afirmó Kehlet en sus conferencias en nuestro Congreso, el control del dolor a través de la analgesia peridural continua es el factor más importante para la reducción del tiempo e intensidad del íleo.

En el trabajo que hoy comentamos, todos menos un paciente expulsaron gases por ano antes de las 48 horas, y el 50\% tuvo una evacuación en el mismo lapso. No se explicita si hubo que suspender la ingesta en los 7 que presentaron náuseas y en los 3 que tuvieron vómitos, pero lo concreto es que el $78 \%$ del universo estudiado fue dado de alta al $4^{\circ}$ día y otro $15 \%$ al $5^{\circ}$, lo que se correlaciona en forma lógica con el dolor postoperatorio, que fue EVA menor de 1 en el 95\% de los pacientes en las primeras 48 horas, y que se incrementó hasta EVA menos de 3 en el día $3^{\circ}$, coincidiendo con el retiro de la analgesia epidural.

En la discusión el Dr. Espíndola compara sus resultados con una serie anterior de su mismo grupo de trabajo y con otras comunicaciones nacionales, apreciándose estadías hospitalarias más breves en este estudio de recuperación acelerada y en la de cirugía laparoscópica del Hospital de la Universidad Católica; ello le permite afirmar algo que compartimos: la recuperación postoperatoria depende de factores como control óptimo del dolor, alimentación y deambulación precoces y uso selectivo (y por tanto restringido) de drenajes y sondajes, más que a la vía de abordaje en sí; de hecho, 2 interesantes estudios de Basse '03 y '05 no demuestran diferencias significativas en la estadía de pacientes sometidos a cirugía de colon abierta versus laparoscópica.

Sr. Presidente, respetados colegas, sólo me resta felicitar al Dr. Leonardo Espíndola por esta con- 
tribución original para nuestro medio, que servirá de modelo para un enfrentamiento más moderno y racional del paciente quirúrgico. Por tanto, me permito recomendar su admisión como Miembro Titular de nuestra Sociedad.

\section{Referencias}

1. Reissman P, Teoh TA, Cohen SM. Is early oral feeding safe after elective colorectal surgery: a prospective randomized trial. Ann Surg 1995; 222: 73-77.

2. Choi J, O'Connell TX. Safe and effective early postoperative feeding and hospital discharge after open colon resection. Am Surgeon 1996; 62: 853-856.

3. Ortiz H, Armendariz P, Yarnoz C. Is early postoperative feeding feasible in elective colon and rectal surgery? Int J Colorectal Dis 1996; 11: 119-121.

4. Hawasi A, Schroder DM, Lloyd LR. Elective conventional colectomy in the era of laparoscopic surgery. Am Surgeon 1996; 62: 589-593.

5. Di Fronzo LA, Cymerman J, O'Connell T. Factors affecting early postoperative feeding following elective open colon resection. Arch Surg 1999; 134: 941-946.

6. Carli F, Phil M, Trudel J. The effect of intraoperative thoracic epidural anesthesia and postoperative analgesia on bowel function after colorectal surgery. Dis Colon Rectum 2001; 44: 1083-1089.

7. Holte K, Kehlet H. Postoperative ileus: a preventable event. Brit J Surg 2000; 87: 1480-1490.

8. Wilmore D, Kehlet H. Management of patients in fast track surgery. Brit Med J 2001; 322: 473-476.

9. Kehlet H, Wilmore D. Multimodal strategies to improve surgical outcome. Am J Surg 2002; 183: 630641.

10. Basse L, Thorbol JE, Lossi K. Colonic surgery with accelerated rehabilitation or conventional care. Ann Surg 2003; 232: 51-57.

11. Basse L, Jakobsen DH, Bardram L. Functional recovery after open versus laparoscopic colonic resection. A randomized, blinded study. Ann Surg 2005; 241: 416423.

Dr. Jaime Contreras $P$. Hospital Clínico San Borja-Arriarán Santiago, Chile. 